\title{
EXPERIENCIAS DE ENGAGEMENT DE APRENDIZAJE DE JÓVENES UNIVERSITARIOS MEXICANOS CON YOUTUBE
}

\section{Learning Engagement Experiences of Higher Education Students in Mexico with YouTube}

\section{Ligia García-Béjar ${ }^{1}$}

ligarcia@up.edu.mx

1 Universidad Panamericana México

Recibido: $21 / 08 / 2020$

Revisado: 4/04/2021

Aprobado: $23 / 04 / 2021$

Preprint: 7/06/2021

Publicado: 15/07/2021

\section{Resumen}

El artículo presenta los resultados de la aplicación de la escala de engagement digital de Mersey, Calder y Malthouse, que mide distintas experiencias de engagement en la utilización de plataformas digitales para conocer cómo los estudiantes universitarios están usando YouTube. Se extrapolaron de la escala las dimensiones educativas que presenta el modelo para hacer un análisis factorial de las dimensiones de engagement con YouTube más presentes entre la población universitaria de licenciatura y posgrado en la Zona Metropolitana de Guadalajara. Los resultados resaltan de manera remarcada cómo los estudiantes mexicanos se identifican con esta red social y presentan experiencias de consumo individuales y sociales, y la usan como una herramienta vital para reforzar sus procesos de aprendizaje autónomos, y cómo la navegación en la plataforma se ha convertido para ellos en un espacio alterno y complementario a sus espacios formales de aprendizaje, más allá de usarla para entretenerse o informarse.

Palabras clave: aprendizaje, engagement, estudiantes universitarios, medios sociales, México, YouTube.

\section{Abstract}

The article presents the results of the engagement with digital media model of Mersey, Calder and Malthouse, which measures different engagement experiences in the use of digital platforms and social media. In order to understand how university students are using YouTube. The educational dimensions presented by the model were extrapolated from the scale to make a factorial analysis of the engagement dimensions among the undergraduate and graduate university population in the Guadalajara, Metropolitan Area, Mexico. The results highlight how Mexican students identify with this social network and present individual and social consumption experiences with this platform, and use it, as a vital tool to control their autonomous learning processes, and how the use of YouTube has become an alternative and complementary space for them to enrich their formal learning spaces, beyond using it for entertainment and information purposes.

Keywords: undergraduate students, learner engagement, social media, Mexico, YouTube. 


\section{Introducción}

El uso de las tecnologías de la información ha acercado a los usuarios a experimentar diferentes tipos de comportamiento y experiencias en distintos ámbitos de la vida, entre ellos el escolar y, en el caso de este artículo, el universitario. La importancia de estudiar la influencia de la tecnología en este nivel educativo se debe al crecimiento de la información digital a disposición de los estudiantes de este nivel y al tiempo que pasan utilizando plataformas en línea, las cuales representan una de las fuentes más comunes para el aprendizaje entre los estudiantes universitarios (Rodríguez \& Fernández, 2017).

YouTube, red social lanzada en 2005 y propiedad de Google, es la plataforma de videos generados por usuarios más grande del mundo (Soukup, 2014). Con un portafolio de contenidos particularmente vasto que incluye musicales, clips de televisión, videos tutoriales o instrucciones, clases en video, juegos en línea y videos educativos, esta red social no solo ha permitido que los espectadores de medios tradicionales se conviertan en generadores de contenido, sino que ha representado una competencia para los medios tradicionales, lo que pone en evidencia la necesidad de las audiencias de interactuar con lo que consumen. Además, la diversidad de su oferta ha abierto la posibilidad de fragmentar contenidos de tal manera que casi cualquier persona puede encontrar algo de su interés en YouTube.

El éxito de dicha plataforma puede entenderse a partir del incremento que ha tenido el consumo de videos en internet en diferentes formatos. De acuerdo a Statista (2019), cada minuto se suben 500 horas de video en YouTube. En el caso de México, ver videos en esa red social es la principal actividad que los usuarios de teléfonos móviles realizan en su dispositivo, por encima de oír contenidos de audio, usar aplicaciones o jugar videojuegos (IFT, 2019). Además, de acuerdo con IAB (2019), después de Facebook y WhatsApp, YouTube es la red social más usada del país, con una penetración del $82 \%$ de la población.

Esta plataforma también se ha posicionado como influenciadora de decisiones en hábitos de vida y consumo, así como de formas alternativas y directas de aprendizaje para las personas (Google, 2017a, 2017b), tema del que se ocupa este artículo. Para Torres-Ramírez et al. (2014), YouTube es una aplicación muy utilizada y valorada por los estudiantes universitarios, sobre todo como complemento para repasar lo que sus profesores les han enseñado de manera presencial.

El principal objetivo de esta investigación es describir si los alumnos generan experiencias de engagement en YouTube y resaltar la percepción que tienen sobre cómo esta plataforma puede contribuir a complementar sus procesos de aprendizaje.

El alcance de este artículo radica en dos aspectos: desde el punto de vista teórico, el engagement emerge como un concepto que ha contribuído de manera importante a entender mejor aquellas experiencias de consumo que aportan más valor a los usuarios de medios digitales, sobre todo tomando en cuenta que los jóvenes son fuertes usuarios de redes sociales. Estas experiencias influyen en su manera de consumir información, entretenimiento y, en el caso de YouTube, aprendizaje, pues al usarla ponen en práctica competencias de autonomía y autorregulación de su propios procesos de aprendizaje más allá de la educación formal. Desde la perspectiva práctica, es importante que los docentes conozcan aquellas experiencias de aprendizaje que esta red social posibilita a los estudiantes, de tal manera que valoren su utilización como alternativa dentro de los recursos de aprendizaje, tomando en cuenta que los alumnos la consideran útil (Tan \& Pearce, 2012). Esta investigación resulta innovadora y relevante para complementar estudios existentes sobre el uso que los jóvenes mexicanos hacen de plataformas de internet, más allá del consumo de información y entretenimiento.

\section{Revisión de la literatura}

\subsection{Utilización educativa de YouTube en la edu- cación universitaria}

Los procesos de aprendizaje de los estudiantes universitarios son cada vez más complejos y requieren 
un elevado grado de motivación (Marcelo, 2019), por lo que cualquier estrategia que contribuya a que se impliquen racional y emocionalmente con su aprendizaje es útil para ayudarles en sus procesos metacognitivos.

La utilización de YouTube como herramienta de aprendizaje es un tema que ha sido explorado para distintos propósitos en la educación superior; en primera instancia, como un respositorio de información, pero más allá de ello, la naturaleza de esta red social permite que los usuarios compartan contenidos y que además intercambien ideas sobre lo que están aprendiendo, lo cual es útil para ellos en cuanto a clarificar y reforzar su entendimiento sobre algún tema.

Los usos educativos de YouTube han sido investigados a nivel superior a partir de su utilidad en distintos ámbitos del conocimiento como Ciencias Computacionales, Medicina, Química y Física (Everson et al., 2013; Lima et al., 2016; Yellepeddi \& Roberson, 2016; Zavalza et al., 2019). En la revisión de literatura de Snelson (2011) se analizaron artículos académicos y ponencias entre los años 2006 y 2011, se descubrió que las áreas de conocimiento que más utilizaban YouTube en la universidad eran Educación, Medicina, Estudios de la Comunicación, Ciencias Políticas y Ciencias Computacionales.

Otro tema investigado sobre la utilización de YouTube en la universidad es la pertinencia de la creación de canales de YouTube por el profesor como complemento de una materia (Rodríguez \& Fernández, 2017). En este punto, para Semich y Copper (2016), un canal educativo también contribuye al desarrollo de competencias digitales para la enseñanza y mejora de los aprendizajes de los alumnos. Aunado a esto, Hrastinski y Aghaee (2012) exploraron el beneficio de esta plataforma como herramienta que contribuye al aprendizaje colaborativo entre los mismos alumnos, sobre todo si forma parte de una estrategia docente planeada.

En lo relacionado al uso potencial de YouTube como apoyo para el aprendizaje, la utilidad que tiene esta herramienta es la facilidad que perciben los alumnos para para usarla con propósitos académicos, debido a que ya están familiarizados con ella (Clifton, 2010). Esta red social ya es usada como herramienta informal para resolver cuestionamientos y dudas sobre la vida diaria, por lo que su incorporación para aprendizajes más formales es prácticamente inmediata. YouTube tiene la ventaja de llegar a los estudiantes en momentos y lugares en los que los profesores no están presentes.

En una investigación empírica, Asselin (2011) descubrió que los alumnos universitarios reportaban obtener aprendizajes significativos a partir de analizar la información consultada en esta red social, incluidos los comentarios de los mismos usuarios. A través de esta herramienta, el profesor puede detectar necesidades de reforzamiento del aprendizaje. Al explorar la utilización de YouTube como recurso para el aprendizaje, Chintalapati y Daruri (2017) descubrieron que las características de esta red social permiten a los estudiantes regular el ritmo el ritmo de su aprendizaje.

Desde las perspectiva del formato, Martínez et al. (2017) destacan que los videos docentes cortos son un material adecuado muy valorado por los estudiantes de las universidades y un acompańamiento muy eficaz de sus clases (Ros-Gálvez \& Rosa-García, 2014), así mismo, influyen en el aprovechamiento académico. Lo anterior se comprobó a partir de analizar la influencia de videos de YouTube en el aprendizaje del idioma francés (Rico et al., 2016). Hablando de valoración por parte de los alumnos, Serrano y Murillo (2018) encontraron en un estudio que esta red social es una herramienta que potencia la motivación cuando los alumnos se dan cuenta que consiguen aprender mejor después de usar o ver contenidos que les ayudaban a entender algún tema.

Finalmente, para utilizar YouTube como recurso educativo, es determinante que el profesor realice una curaduría de los contenidos, procesos de filtrado y recomendaciones a los alumnos, pues la elección de videos de mala calidad o de origen engañoso puede ser contraproducente en la percepción de los alumnos (Almobarraz, 2018). La utilización de material de YouTube en clases es pertinente en la 
medida en que el profesor sugiere a los alumnos una curaduría de contenidos que puedan serles de apoyo o utilidad (Moghavvemi et al., 2018). En conclusión, la intervención planeada en esta red social permite que profesores y maestros tengan su propia biblioteca virtual de videos para conformar una comunidad de aprendizaje en torno a los contenidos seleccionados o creados por su propia autoría (Ramírez-Ochoa, 2014).

\subsection{Engagement con redes sociales y su implica- ción educativa}

Los antecedentes de los estudios sobre el engagement educativo surgen de la premisa de que los entornos escolares requieren climas positivos para que el aprendizaje suceda, y también es necesario sentirse parte de una comunidad para lograrlo (Taylor \& Parsons, 2011). El engagement puede definirse como la comprensión de la participación de estudiantes en su contexto escolar académico y no académico. Se puede categorizar en varias dimensiones: como engagement escolar (Fredericks et al., 2004), engagement académico (Libby, 2004), engagement de estudiantes (Chapman, 2003; Yazzie-Mintz, 2007) y engagement de los estudiantes con la escuela (Klem \& Connell, 2004).

La importancia de este concepto se hizo patente a partir de que se buscaba identificar la razón por la cual los estudiantes no se implicaban en clase y con la escuela, en general (Appleton et al., 2008). Lo anterior es relevante porque el concepto de engagement en individual implica que la actuación de las personas tiene siempre un compotente de conducta individual o social y componentes emocionales y afectivos (Audas \& Willms, 2001). Otros constructos asociados al concepto de engagement son la autorregulación, la formulación de metas y el tiempo que se está dispuesto a pasar haciendo una tarea (Fredericks et al., 2004), en este caso, educativa. Reschly $\&$ Christenson (2006a, 2006b) tipificaron la existencia de cuatro categorías de engagement de los estudiantes: académico, de comportamiento, cognitivo y psicológico.

Las investigaciones más recientes sobre el engagement académico han versado sobre ayudar a los estudiantes a entender las mejores maneras en las que pueden aprender con independencia del tema a estudiar, el autoconocimiento de los estudiantes sobre sus modos de estudiar, explorar sus intereses académicos y desarrollar un aprendizaje más autónomo (Dunleavy \& Milton, 2009; Parsons \& Taylor, 2011).

Las redes sociales son una ruta alternativa para despertar interés de los alumnos con contenido complementario sobre lo que están aprendiendo, aprovechando el hecho de que ya usan las redes sociales y les gusta usarlas (Johntson et al., 2018). Arndt y Wore (2108) afirman que YouTube genera engagement pues propicia aprendizaje incidental, entendiendo por este el que sucede cuando los alumnos le prestan atención a algo de manera inmediata. Si la atención de los alumnos se sostiene, este aprendizaje se vuelve intencional.

El constructo de engagement a analizar en este artículo es el académico, particularmente por qué YouTube genera interés hacia el aprendizaje y cómo puede contribuir a generar una mayor autonomía en los procesos de aprendizaje.

Estudiar el engagement de aprendizaje en plataformas digitales tiene utilidad práctica importante pues ayuda a comprender que la conexión e implicación psicológica de los estudiantes con sus estudios y con la escuela puede jugar un rol importante para despertar una mayor motivación y participación en sus estudios.

\subsection{Modelo utilizado para medir engagement académico con YouTube}

El modelo seleccionado para hacer esta descripción es el modelo de engagement con redes sociales de (2010). A través de este modelo también se pretende saber más de las creencias, pensamientos e ideas que los estudiantes tienen sobre sus experiencias de engagement con YouTube, a partir de la percepción que manifiestan los participantes, a través de una colección de experiencias que hacen alusión a las creencias e ideas que consideran sobre cómo las redes sociales que consumen encajan en diversos aspectos de su vida (2010). Atendiendo a la pertinencia de utilizar 
el engagement para comprender el consumo de YouTube, el desarrollo del modelo de Mersey, Malthouse y Calder que será usado en esta investigación tiene sus antecedentes en la perspectiva de usos y gratificaciones (Katz \& Gurevitch, 1973), la tipología de usos y gratificaciones desarrollada por McQuail (1987), y la adaptación a usuarios interactivos de la misma (Ruggiero, 2000).

Este es un modelo aplicado específicamente a cómo sucede el consumo de redes sociales: por qué tienen sentido para el usuario, cómo se vincula el usuario con una comunidad más amplia, por qué las redes son útiles para las personas y por qué hay experiencias de aprendizaje al utilizarlas. De ahí la pertinencia de elegirlo para estudiar el consumo de YouTube.

Para Mersey et al. (2010), las experiencias de los usuarios tienen una dimensión personal y otra social-interactiva. La personal hace referencia al conjunto de manifestaciones que las personas suelen tener con medios tradicionales; por otro lado, la parte social-interactiva es más específica del consumo de medios digitales, específicamente en redes sociales, y se hace visible a través de comportamientos que ponen en evidencia que el usuario está utilizando estas herramientas para socializar con otras personas. En general, a través del consumo de medios de comunicación las personas buscan estimulación, inspiración, interacción con otras personas a propósito del contenido, afirmación de la autoestima y sensaciones intrínsecas de gozo o satisfacción. En las experiencias de engagement social-interactivo siempre están incluidas las personales. En complementariedad, el compromiso social-interactivo está motivado por la relevancia social y una experiencia de compromiso y de comunidad mucho más amplia, por eso se valoran en estas dimensiones aspectos relativos a la participación con otros y la socialización. El sentido de utilidad y de gozo intrínseco también están presentes en estas experiencias de implicación.

Estas experiencias, a su vez, implican dimensiones diversas de engagement, mediante las cuales las personas expresan lo que entienden sobre sus comportamientos, razonamientos y sentimientos en relación a su consumo de medios de comunicación. Las dimensiones comprenden: experiencias de estimulación e inspiración, de facilitación social, de temporalidad, de autoestima y conciencia cívica, de entretenimiento intrínseco, de utilidad, de participación y socialización y de sentido de comunidad. De acuerdo a este modelo, algunas dimensiones comprenden experiencias de engagement individual y otras, experiencias sociales e interactivas. A partir de las 37 experiencias concretas (ver Figura 1), se genera el cuestionario de medición seleccionado para este trabajo de investigación.

\section{Método}

Para detectar el engagement académico de estudiantes universitarios con YouTube se seleccionó un diseño cuantitativo para obtener la información pertinente sobre cómo el consumo de esta plataforma impacta en los alumnos universitarios. Con este diseño se pretendía: a) utilizar una herramienta válida para medir experiencias de engagement con YouTube y, b) obtener más información sobre las dimensiones que impacta en el ámbito académico del estudiante.

Esta es una investigación empírica-analítica (Arnal et al., 1996) con un diseño descriptivo, no experimental y transeccional (Hernández-Sampieri, 2014), pues busca especificar las propiedades del engagement entre la población universitaria de la Zona Metropolitana de Guadalajara, en México. Es descriptiva y no experimental, porque no hay intención de manipular deliberadamente las variables a investigar, sino observar un fenómeno tal y como aparece en su entorno natural.

Se considera que este tipo de diseño es acertado, ya que para comprender la interacción real de las personas con las plataformas audiovisuales es apropiado conocer la percepción de los usuarios y no el diseño de un experimento para observar o manipular el consumo de los usuarios. Es transeccional porque se recolectaron datos en un momento único, lo que permite describir las variables a analizar, su incidencia e interrelación en un momento específico. Finalmente, se ha elegido este diseño con el propósito de avanzar en el conocimiento del engagement educativo del alumno universitario de la Zona Metropolitana de Guadalajara. 


\section{Figura 1. Modelo de engagement}

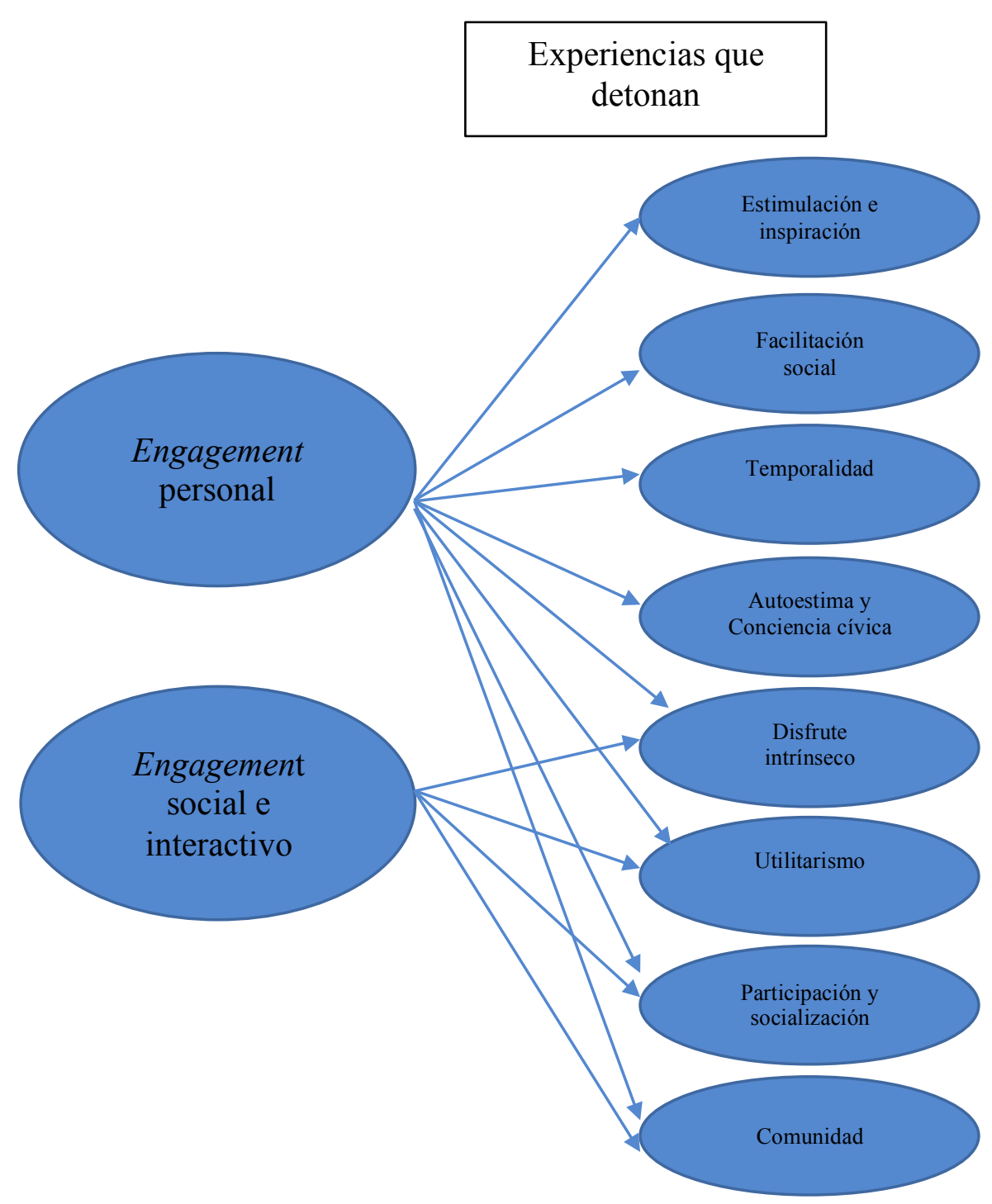

Nota: Elaboración a partir del modelo de Mersey, Malthouse y Calder, 2010.

El instrumento cuantitativo seleccionado para la recolección de datos es un cuestionario electrónico y autoadministrado grupal e individualmente, con preguntas cerradas de respuesta única y escalamiento estimativo tipo Likert. Los ítems fueron adaptados a partir del modelo original de Mersey et al. (2010), contextualizándolos al uso de YouTube con una escala estimativa en la que los usuarios pudieran señalar el nivel de acuerdo o desacuerdo sobre las 37 experiencias de engagement identificadas en el mismo. Entre estas se analizaron específicamente las correspondientes a las dimensiones de aprendizaje. Se añadieron otras preguntas que permitieran tener información descriptiva sobre la muestra seleccionada.

La escala seleccionada fue del 1 al 7 , en la que 1 era "muy en desacuerdo" y 7 "muy de acuerdo", más la opción 8 de "no aplica" en el caso de que la percepción sobre la presencia de esa experiencia de engagement es nula. Este rango de 7 entre la respuesta más afirmativa y la más negativa se hizo para lograr 
un mayor nivel de discriminación de opciones y porque en ejemplos previos de aplicación del modelo se tomó también esa decisión.

La investigación se llevó a cabo en la Zona Metropolitana de Guadalajara (ZMG), que además de ser la zona conurbada más grande de México por número de habitantes, es una ciudad que concentra una población universitaria importante dentro de México, capta estudiantes locales y de una parte importante del país: la zona centro, el bajío, el noreste y noroeste de México, y cuenta con la segunda universidad pública más grande del país y por lo menos 8 universidades registradas ante las autoridades educativas del país (ANUIES, 2019).

El universo de este estudio son jóvenes universitarios que viven en la Zona Metropolitana de Guadalajara y nacieron entre el ańo 1981 y el año 2000; dicha población es de $N=1,347,028$ de acuerdo al IIEG (2018). La muestra fue no probabilística por racimos y seleccionada a partir de la distribución muestral por proporciones, se utilizó una probabilidad de acierto (p) de 0.5 para determinar el tamańo de la muestra, con un nivel de confianza (z) del $95 \%=1.96 \%$ y un margen de error (e) del $5 \%$. Lo anterior dio por resultado una muestra (n) de 384 jóvenes; se realizó un muestreo por conveniencia y se logró llegar a una muestra de 574 encuestas, lo cual bajó el margen de error (e) al 4.09\%. Aunque el cuestionario fue contestado por 762, después de tomar decisiones respecto al alcance de la muestra se tuvieron que invalidar algunos cuestionarios, otros fueron excluidos porque no habían pasado los filtros (respondieron estando en otra ciudad o no tenían la edad indicada, por ejemplo) o no habían terminado de contestar la encuesta.

Para obtener acceso a la muestra, se seleccionó un público universitario y se envió la encuesta a través de correos electrónicos de los alumnos de algunas de las principales universidades de la Zona Metropolitana de Guadalajara, se contactó a profesores y se visitó físicamente 4 de las 5 universidades para asegurar la calidad de la aplicación y tener un margen muestral más amplio. Se invitó a participar a las personas en salones de clase con el permiso de los profesores y también se invitaba a participar en los jardines. Por esta razón, las visitas en vivo fueron más efectivas que los envíos en correos electrónicos. En este contexto también se tuvo acceso a alumnos y aulas de licenciatura y posgrados.

\section{Resultados}

Luego de la recolección de la información, la plataforma QuestionPro proporcionó una base de datos en Excel, se revisó la codificación y se limpiaron los datos, después se transfirieron al programa de análisis estadístico SPSS para ser procesados. Se hicieron diversos análisis descriptivos, así como la relación entre algunas variables, sobre todo relacionadas con el consumo de las plataformas elegidas: los tiempos de consumo, las diferencias de edad y sexo respecto al consumo y las formas de engagement académico encontradas de acuerdo al modelo de Mersey et al. (2010).

Los 574 participantes viven en alguno de los $5 \mathrm{mu}$ nicipios pertenecientes a la Zona Metropolitana de Guadalajara: Guadalajara, Zapopan, Tonalá, Tlaquepaque y Tlajomulco y otros municipios aledaños más pequeños. En la ZMG, los municipios con mayor número de habitantes son Guadalajara y Zapopan, con 1.53 y 1.36 millones de habitantes (IIEG, 2018), respectivamente.

Respecto a los demás datos descriptivos, la encuesta fue respondida por 365 mujeres y 209 hombres, correspondiendo al $63.6 \%$ y al $36.4 \%$, respectivamente. Esta distribución es relativamente coherente con el hecho de que, de acuerdo al IIEG (2018), en la zona metropolitana de Guadalajara hay una mayor población de mujeres que de hombres, particularmente en el municipio de Guadalajara. También tiene que ver con la población estudiantil a la que se encuestó, que fueron de áreas de ciencias sociales y de empresariales y ciencias económicas, cuya población femenina es mayor a la masculina. Sería importante poder buscar una media más proporcional entre ambos sexos. La distribución de la muestra quedó más representada por personas de entre 18 y 23 ańos, no obstante, hay representación 
de participantes de todas las edades del rango seleccionado. Este porcentaje influirá en los demás resultados, que serán más representativos para algunas franjas de edad. Este resultado se obtuvo por la recepción que tuvo la respuesta al cuestionario entre población universitaria, así como la facilidad de llegar a ese clúster. En la muestra, el 56.3\% ha terminado el bachillerato, el $34.7 \%$ ha terminado una licenciatura, y el 9.1\% cuenta con algún posgrado. La muestra del estudio es coherente con la información sobre la penetración de YouTube en la población mexicana. Casi el $80 \%$ de los jóvenes universitarios consumen YouTube (ver Tabla 1).

La información sobre las pantallas que utilizan los estudiantes universitarios para ver Youtube es relevante desde la perspectiva educativa, pues otorga información valiosa sobre cómo los jóvenes están utilizando sus dispositivos en la vida diaria y, por tanto, para sus estudios (ver Tabla 2), así mismo, el tiempo que los estudiantes pasan en la plataforma, diferenciando días hábiles y fines de semana (ver Tabla 3).

Tabla 1. Utilización de YouTube entre la población encuestada

\begin{tabular}{l|l|r|r|r|r}
\hline \multicolumn{2}{l|}{} & Frecuencia & Porcentaje & $\begin{array}{c}\text { Porcentaje } \\
\text { válido }\end{array}$ & $\begin{array}{r}\text { Porcentaje } \\
\text { acumulado }\end{array}$ \\
\hline \multirow{2}{*}{ Válido } & Sí & 452 & 78.7 & 80.1 & 80.1 \\
\cline { 2 - 6 } & No & 112 & 19.3 & 19.7 & 99.8 \\
\cline { 2 - 6 } & Total & 564 & 98.3 & 100.0 & \\
\hline Perdidos & Sistema & 10 & 1.7 & & \\
\hline \multicolumn{2}{l|}{ Total } & 574 & 100 & & \\
\hline
\end{tabular}

Nota: Elaboración propia.

Tabla 2. Distribución de consumo de YouTube por pantallas

\begin{tabular}{lccccc}
\hline Plataforma & Teléfono móvil & Laptop & Tableta & Smart TV & No consume \\
\hline YouTube & $69 \%$ & $22 \%$ & $5.4 \%$ & $4.4 \%$ & $2.4 \%$ \\
\hline
\end{tabular}

Nota: Elaboración propia.

Tabla 3. Tiempo de consumo de YouTube entre semana y en fin de semana

\begin{tabular}{lccccc}
\hline Pregunta & Sexo & N & Media & $\begin{array}{c}\text { Desviación } \\
\text { estándar }\end{array}$ & Media de error estándar \\
\hline $\begin{array}{l}\text { Min. al día que se pasa } \\
\text { en YouTube }\end{array}$ & $\mathrm{M}$ & 365 & 99.46 & 199.71 & 10.45 \\
\cline { 2 - 6 } $\begin{array}{l}\text { Min. al día que se pasa } \\
\text { en YouTube en fin de }\end{array}$ & $\mathrm{H}$ & 208 & 112.78 & 162.02 & 11.23 \\
\cline { 2 - 6 } \begin{tabular}{l} 
semana \\
\cline { 2 - 6 }
\end{tabular} & $\mathrm{H}$ & 208 & 96.36 & 177.69 & 9.30 \\
\hline
\end{tabular}

Nota: Elaboración propia. 
En lo relativo al corazón de este estudio sobre el engagement desde el modelo de Mersey, Malthouse y Calder, se determinó aplicar los siguientes análisis: alfa de Cronbach, análisis descriptivos y factoriales, basados en los análisis observados a partir de la revisión de literatura. Se utilizó este tipo de análisis dado que los constructos estudiados para cada plataforma son muchos y era necesario buscar la mayor síntesis y agrupación de los componentes principales para analizarla con mayor pertinencia.

\section{Tabla 4. Confiabilidad del instrumento para medir engagement con YouTube}

\begin{tabular}{cc}
\hline Alfa de Cronbach & N de elementos \\
\hline 0.970 & 37 \\
\hline
\end{tabular}

Nota: Elaboración propia.
La prueba de alfa de Cronbach permitió determinar una consistencia interna alta de cada una de los constructos analizados. En este sentido, los resultados nos permiten determinar que las experiencias medidas para cada plataforma arrojan resultados confiables, pues se ubican en el 0.970 , es decir, el modelo de Mersey, Calder y Malthouse es útil para medir la experiencia de engagement de los usuarios.

Luego del análisis de fiabilidad se hicieron análisis descriptivos para conocer las medias y la desviación estándar de los atributos de engagement (ver Tabla 5). Para representar estos resultados se tomó la decisión de elegir únicamente aquellos que tenían una medía superior a 3.70, tomando en cuenta que la escala fue del 1 al 7, y para los atributos más bajos por plataforma fueron elegidos los que presentaron una media por debajo de 3 .

\section{Tabla 5. Atributos más altos de engagement con YouTube}

\begin{tabular}{lcc}
\hline \multicolumn{1}{c}{ Atributo } & Media & \multicolumn{1}{c}{$\begin{array}{c}\text { Desviación } \\
\text { estándar }\end{array}$} \\
\hline $\begin{array}{l}\text { Uso YouTube como parte importante del contenido de entretenimiento que con- } \\
\text { sumo }\end{array}$ & 4.05 & 1.994 \\
\hline $\begin{array}{l}\text { Creo que los usuarios de YouTube podemos aprender del contenido que encontra- } \\
\text { mos en la plataforma }\end{array}$ & 4.34 \\
\hline Me he llegado a interesar en temas que he visto en YouTube & 4.33 & 2.155 \\
\hline El contenido de YouTube me hace pensar de nuevas maneras & 3.89 \\
\hline Me gusta ver YouTube cuando estoy tomando un descanso o comiendo & 4.10 \\
\hline Ver YouTube me provoca satisfacción & 3.81 \\
\hline $\begin{array}{l}\text { YouTube es uno de los sitios de internet que reviso cada vez que estoy surfeando } \\
\text { en la red }\end{array}$ & 4.09 \\
\hline El contenido de YouTube me ha dado temas de conversación & 2.044 \\
\hline $\begin{array}{l}\text { El contenido de YouTube me ha servido para argumentar o discutir sobre algún } \\
\text { tema }\end{array}$ & 3.902 \\
\hline El contenido de YouTube me estimula a pensar sobre diferentes temas & 2.096 \\
\hline Me he descubierto hablando sobre YouTube o sobre contenido de YouTube en con- & 3.911 \\
\hline \begin{tabular}{l} 
versaciones con otras personas \\
\hline
\end{tabular} & 4.91 \\
\hline
\end{tabular}

Nota: Elaboración propia. 
En el caso de YouTube, se muestran algunas constantes en las dimensiones de estimulación, inspiración y disfrute, aunque hay una fragmentación mayor de dimensiones, pues se muestran variables de la dimensión de socialización y facilitación, utilitarismo y, sobre todo, comunidad. Esta misma fragmentación se encuentra en los atributos que manifiestan una conexión más baja. Esto puede implicar que el uso de YouTube es menos uniforme, dada la diferenciación y la diversidad de sus contenidos, producidos tanto por los usuarios como los contenidos distribuidos por productores y empresas de comunicación. Lo que sí se puede apreciar en los resultados es que hay una manifestación de uso de YouTube para el enriquecimiento o aprendizaje tanto personal como social.

Posteriormente se hizo un análisis factorial; se utilizó el método de rotación que minimiza el número de factores necesarios para explicar cada variable, en este caso atributos de engagement, pues hay que tomar en cuenta que son muchos los atributos investigados sobre cada constructo de la encuesta y era necesario encontrar una forma clara de conocer las dimensiones con sus atributos específicos. Este tipo de análisis permitió corroborar cuáles eran las formas de uso más significativo, tomando en cuenta que se considera una variable de correlación alta aquella que está muy cercana o por encima del 0.7. Este análisis también ayuda a reconfirmar la validez de los constructos en relación a lo que estamos midiendo: engagement con cada una de las plataformas en sus distintas dimensiones.

El análisis factorial permite hacer una identificación más fina de las experiencias más valoradas por los usuarios, los cuales consideran que usan YouTube por razones de socialización y participación, sentido de comunidad y después de utilitarismo (ver Tabla 6), lo cual demuestra la naturaleza más participativa e incluyente de los usuarios de YouTube, por ser una red social cuyos contenidos son construidos por los mismos usuarios. En este sentido se logra conectar con contenidos que son atractivos y relevantes para ellos. A través de este análisis los usuarios manifiestan que la conexión con esta plataforma va más allá del entretenimiento e implica oportunidades de aprendizaje.

Este análisis permite destacar experiencias de engagement relacionadas con la inspiración y la estimulación del pensamiento, y como herramienta para hablar con otras personas sobre un contenido específico. El contenido que consumen en esta herramienta incluso les sirve para la toma de decisiones. YouTube también es una red social utilizada para desconectarse y relajarse; este elemento se corrobora al comprobar el tiempo que pasan los jóvenes usando YouTube. Es una red social para compartir, por tanto, la interacción de los usuarios y la producción comunitaria son elementos esenciales. Hay un sentido de comunidad usando esta plataforma, y se asemeja de alguna manera a los procesos de enseñanza-aprendizaje socializado en las aulas de clase.

\section{Conclusiones}

Esta investigación amplía la comprensión sobre cómo los estudiantes universitarios están utilizando YouTube y qué los motiva a estar en esta red social y a contribuir no solo en el consumo del contenido sino en la producción del mismo.

A partir de los resultados obtenidos en este estudio, se ha podido establecer que los jóvenes mexicanos universitarios están pasando tiempo considerable en sus pantallas, consumiendo plataformas audiovisuales como YouTube. Tomando en cuenta que los autores del modelo utilizado identifican una colección más vasta de experiencias, la muestra encuestada determina muy claramente las dimensiones con las que identifica su consumo de la red social elegida; dicho consumo se conecta con otros aspectos de su vida, por tanto, se conecta también con su experiencia de estudio, siendo esta quizá la actividad prioritaria en su vida. La edad es un factor relevante para el tipo de consumo, el tiempo que se consume y para la conciencia sobre el engagement que se tiene con el consumo de YouTube. Este elemento ayuda a entender no solo que los alumnos busquen en esta plataforma información que necesitan, también explica por qué los profesores la están utilizando, tal y como se expone en la literatura. 


\begin{tabular}{|c|c|c|c|c|c|}
\hline \multirow[t]{2}{*}{ Atributo } & \multicolumn{5}{|c|}{ Componente } \\
\hline & 1 & 2 & 3 & 4 & 5 \\
\hline Me he llegado a interesar en temas que he visto en YouTube & 0.730 & 0.347 & 0.275 & 0.004 & 0.078 \\
\hline $\begin{array}{l}\text { Creo que los usuarios de YouTube podemos aprender del } \\
\text { contenido que encontramos en la plataforma }\end{array}$ & 0.716 & 0.339 & 0.285 & 0.013 & 0.083 \\
\hline $\begin{array}{l}\text { En YouTube se pueden encontrar formas de mejorar en } \\
\text { distintos aspectos }\end{array}$ & 0.665 & 0.292 & 0.332 & 0.169 & 0.068 \\
\hline $\begin{array}{l}\text { Me he descubierto dando algún consejo a alguien a partir del } \\
\text { contenido que he visto en YouTube }\end{array}$ & 0.664 & 0.201 & 0.306 & 0.272 & 0.170 \\
\hline $\begin{array}{l}\text { YouTube facilita que los usuarios del mismo interactuemos y } \\
\text { demos retroalimentación sobre su contenido }\end{array}$ & 0.654 & 0.211 & 0.250 & 0.141 & 0.230 \\
\hline $\begin{array}{l}\text { El uso de YouTube me da pie a socializar sobre diferentes } \\
\text { temas }\end{array}$ & 0.639 & 0.354 & 0.263 & 0.271 & 0.140 \\
\hline $\begin{array}{l}\text { El contenido de YouTube me ha provisto de información para } \\
\text { tomar decisiones relevantes }\end{array}$ & 0.624 & 0.121 & 0.350 & 0.292 & 0.127 \\
\hline Ver YouTube me relaja bastante & 0.387 & 0.743 & 0.162 & 0.142 & 0.057 \\
\hline Ver YouTube me provoca satisfacción & 0.409 & 0.736 & 0.179 & 0.162 & -0.006 \\
\hline $\begin{array}{l}\text { Ver YouTube mejora mi estado anímico, me hace sentir más } \\
\text { contento }\end{array}$ & 0.421 & 0.732 & 0.157 & 0.196 & 0.001 \\
\hline $\begin{array}{l}\text { Me gusta ver YouTube cuando estoy tomando un descanso } \\
\text { o comiendo }\end{array}$ & 0.295 & 0.711 & 0.166 & -0.014 & 0.194 \\
\hline $\begin{array}{l}\text { Uso YouTube como parte importante del contenido de } \\
\text { entretenimiento que consumo }\end{array}$ & 0.216 & 0.698 & 0.372 & 0.135 & 0.249 \\
\hline Ver YouTube se ha convertido en parte de mi rutina & 0.152 & 0.688 & 0.385 & 0.138 & 0.271 \\
\hline $\begin{array}{l}\text { YouTube uno de los sitios de internet que reviso cada vez } \\
\text { que estoy surfeando en la red }\end{array}$ & 0.146 & 0.686 & 0.398 & 0.055 & 0.251 \\
\hline $\begin{array}{l}\text { El contenido de YouTube me hace pensar de nuevas } \\
\text { maneras }\end{array}$ & 0.280 & 0.237 & 0.789 & 0.147 & 0.134 \\
\hline $\begin{array}{l}\text { El contenido de YouTube me estimula a pensar sobre } \\
\text { diferentes temas }\end{array}$ & 0.334 & 0.275 & 0.761 & 0.126 & 0.068 \\
\hline El contenido de YouTube inspira aspectos de mi propia vida & 0.161 & 0.277 & 0.734 & 0.199 & 0.184 \\
\hline $\begin{array}{l}\text { El contenido que he visto en YouTube me ha hecho } \\
\text { reflexionar con profundidad }\end{array}$ & 0.380 & 0.170 & 0.702 & 0.175 & 0.103 \\
\hline $\begin{array}{l}\text { El contenido de YouTube me hace una persona más } \\
\text { interesante }\end{array}$ & 0.231 & 0.180 & 0.614 & 0.400 & 0.063 \\
\hline $\begin{array}{l}\text { En algún momento he usado contenido visto en YouTube } \\
\text { para argumentar o discutir sobre algún tema }\end{array}$ & 0.444 & 0.241 & 0.610 & 0.160 & 0.161 \\
\hline
\end{tabular}


Otro hallazgo importante de esta investigación es que es posible utilizar modelos de engagement para reconocer el vínculo de las personas con las plataformas y lo contenidos que consumen. Autores como Bergillos (2017) consideran que los usuarios de medios digitales suelen tener más conciencia de su consumo mediático. En un entorno de alta fragmentación de contenidos, las personas otorgan atención y tiempo a aquello que les genera valor. Los hallazgos de este estudio sugieren que los docentes podrían conducir de forma más dirigida a los alumnos en la utilización de contenidos valiosos de YouTube, aprovechando que ellos ya detectan la utilidad de esta red social como herramienta de aprendizaje. La utilización de YouTube puede aportar elementos al autoconocimiento que los alumnos tienen sobre sí mismos y sus limitaciones de aprendizaje, por tanto, buscan contenidos que les ayuden a comprender o confirmar aprendizajes.

YouTube genera engagement por su naturaleza social y los estudiantes expresan a través de distintas dimensiones que es una fuente de mejora y es utilizada como recurso de aprendizaje y educativo. Esta disposición de utilización de la herramienta también podría significar que existe una inclinación por esta herramienta en cuanto descubren en ella un sentido de utilidad al poder repasar contenidos y practicar lo que están aprendiendo en las aulas y, por tanto, reforzar su aprendizaje.

Esta red social es percibida no solo como una herramienta útil, también es una forma en la que se conectan con otros, por lo que usarla implica también compartir con otros lo que saben o lo que han aprendido, aunque este aprendizaje no necesariamente puede tener una conexión directa con su actividad como estudiantes, ya que también puede tener una faceta de sentido práctico para su vida. Finalmente, la disposición que tienen los estudiantes hacia esta red social puede ser potenciada y utilizada positivamente en el contexto universitario y sumar a otros recursos que los profesores utilizan dentro de sus materias.

El estudio presentado contribuye a la conversación sobre la importancia de que los profesores utilicen herramientas que llamen la atención de los alumnos, pero no solo porque las usan mucho, también porque justo a partir de esa familiaridad con la plataforma se pueda enseñar y mostrar a los alumnos maneras de utilizar de forma más pertinente el tiempo que pasan en internet y cómo ese tiempo puede ser productivo y sumar de manera estratégica a sus propósitos de aprendizaje. Además, el uso de una plataforma como YouTube tiene el potencial de apoyar el desarrollo de competencias de autogestión y autoaprendizaje.

Finalmente, la mera evidencia de que esta red social ha ganado relevancia como espacio de aprendizaje es llamativa y representa un tema que los profesores y las universidades deberían estudiar, incluso para determinar estrategias específicas entre la comunidad educativa sobre cómo explotar las posibilidades de la herramienta en clases y ayudar a los alumnos a que la utilicen como un método de aprendizaje de forma más estratégica, con conducción docente y una curaduría planeada para la selección y producción de contenidos. Cabe añadir que la herramienta no complementa el aprendizaje del alumno por sí sola. YouTube es útil en la medida en que el alumno se esfuerza por profundizar en algún contenido, por practicar más algo que ya ha comprendido o buscar contenido que le ayude a dar contexto a algo que está aprendiendo. Por tanto, el papel del profesor es estimular la discusión que cumpla con este objetivo.

Esta investigación pretende dar de inicio una guía para profesores, presenta evidencia sobre cómo los estudiantes universitarios están utilizando YouTube y corrobora que la usan para promover situaciones de aprendizaje, por lo cual podría completarse utilizando información cualitativa de parte de los estudiantes en la que puedan expresar con mayor detalle cómo están utilizando YouTube, partiendo de las experiencias de engagement identificadas en este trabajo.

\section{Referencias bibliográficas}

Almobarraz, A. (2018), Utilization of YouTube as an information resource to support university courses. The Electronic Library, 36(1), 71-81. https://doi.org/10.1108/EL-04-2016-0087 
Asociación Mexicana de Instituciones de Educación Superior ANUIES (2019). Instituciones de Educación Superior. https://bit.ly/2Pi3xtG

Appleton, J. J., Christenson, S. L., Kim, D., \& Reschly, A. L. (2006). Measuring cognitive and psychological engagement: Validation of the Student Engagement Instrument. Journal of School Psychology, 44(5), 427-445.

https://doi.org/10.1016/j.jsp.2006.04.002

Audas, R., \& Willms, J. D. (2001). Engagement and dropping out of school: A life course perspective. Human Resources and Social Development Canada. https://bit.ly/2PfHyn7

Arnal, J., Del Rincón, D., \& Latorre, A. (1992). Investigación educativa. Barcelona: Labor.

Asselin, M., Dobson, T., Meyers, E., Teixiera, C., \& Ham, L.( 2011). Learning from YouTube: an analysis of information literacy in user discourse. En Proceedings of the 2011 iConference (iConference '11). Association for Computing Machinery, New York, NY. https://doi.org/10.1145/1940761.1940851

Bergillos, D. I. (2018). ¿Dos caras de la misma moneda?: una reflexión sobre la relación entre engagement y participación en medios. Comunicación y Hombre, (14), 121-136.

https://r.issu.edu.do/l?l=10554KfL

Chapman, E. (2003). Alternative Approaches to Assessing Student Engagement Rates. Practical Assessment, Research \& Evaluation, 8(13), 1-10.

https://bit.ly/3fsXgGl

Chintalapati, N., \& Daruri, V. S. K. (2017). Examining the use of YouTube as a Learning Resource in higher education: Scale development and validation of TAM model. Telematics and Informatics, 34(6), 853860. https://doi.org/10.1016/j.tele.2016.08.008

Clifton, A., \& Mann, C. (2011). Can YouTube enhance student nurse learning? Nurse education today, 31(4), 311-313. shorturl.at/xB058

Domínguez, C. G., \& Murillo-Estepa, P. (2018). La práctica docente mediada con tecnologías: YouTube como herramienta de aprendizaje en educación superior. Foro educacional, (31), 11-29.

Dunleavy, J. y Milton, P. (2009). What did you do in school today? Exploring the Concept of Student Engagement and its Implications for Teaching and Learning in Canada. Toronto: Canadian Education Association. https://bit.ly/2BQ1dHf

Everson, M., Gundlach, E., \& Miller, J. (2013). Social media and the introductory statistics course. Computers in Human Behavior, 29(5), A69-A81. https://doi.org/10.1016/j.chb.2012.12.033

Fredericks, J. A., Blumenfeld, P. C., \& Paris, A. H. (2004). School engagement: Potential of the concept, state of the evidence. Review of Educational Research, 74, 59-109.

https://doi.org/10.3102/00346543074001059

Garza, J. F. (2017). Análisis de las reproducciones de videos en un canal educativo en YouTube. Memorias del Encuentro Internacional de Educación a Distancia, 5(5).

Google (2017). ¿Cómo ven YouTube en México? https://bit.ly/30ihKNm

Google (2017). ¿Por qué los mexicanos eligen YouTube para ver videos? https://bit.ly/33cQ7Y6

Hernández-Sampieri, R., Fernández-Collado, C., \& Baptista-Lucio, P. (2014). Metodología de la investigación (Vol. 3). México: McGraw-Hill.

Hrastinski, S., \& Aghaee, N.M. (2012). How are campus students using social media to support their studies? An explorative interview study. Education and Information Technologies, 17, 451-464. https://doi.org/10.1007/s10639-011-9169-5

Interactive Association Bureau IAB. (2019). Estudio de consumo de medios y dispositivos entre internautas mexicanos. https://bit.ly/3182Sk1

Instituto Federal de Telecomunicaciones IFT (2019). Encuesta de usuarios de servicios de telecomunicaciones. https://bit.ly/3k278u2

IIEG (2018) Población de Jalisco 2018. https://bit.ly/33fiQLS 
Johnston, A. N., Barton, M. J., Williams-Pritchard, G. A., \& Todorovic, M. (2018). Youtube for millennial nursing students; using internet technology to support student engagement with bioscience. Nurse education in practice, 31, 151-155.

https://doi.org/10.1016/j.nepr.2018.06.002

Katz, E., Blumler, J. G., \& Gurevitch, M. (1973). Uses and gratifications research. The Public Opinion Quarterly, 37(4), 509-523.

Klem, A. M., \& Connell, J. P. (2004). Relationships matter: Linking teacher support to student engagement and achievement. Journal of School Health, 74, 262-273.

https://doi.org/10.1111/j.1746-1561.2004.tb08283.x

Lima, R. M., Rangel, F., Guimarães, M., \& Marcelino, V. (2016). Diabetes mellitus: Generating issues for the teaching of biochemistry. Journal of Biochemistry Education, 14(2), 15.

https://doi.org/10.16923/reb.v14i2.668

Libby, H. P. (2004). Measuring students' relationship to school: Attachment, bonding, connectedness, and engagement. Journal of School Health, 74, 274-283.

McQuail, D. (1987). Mass Communication Theory, an Introduction. London: Sage Publications.

Marcelo, C., \& Rijo, D. (2019). Aprendizaje autorregulado de estudiantes universitarios: Los usos de las tecnologías digitales. Revista Caribeña de Investigación Educativa (RECIE), 3(1), 62-81.

https://doi.org/10.32541/recie.2017.v1i1.pp70-82

Martínez, Á. M., Gálvez, A. R., \& García, A. R. (2017). Difusión de videos académicos: Rendimiento de las universidades españolas en YouTube. En Investigaciones de Economía de la Educación (pp. 515527). Asociación de Economía de la Educación.

Mersey, R. D., Malthouse E., \&. Calder B. (2010). Engagement with Online Media. Journal of Media Business Studies, 7(2), 39-56.

https://doi.org/10.1080/16522354.2010.11073506

Moghavvemi, S., Sulaiman, A., Jaafar, N. I., \& Kasem, N. (2018). Social media as a complementary learning tool for teaching and learning: The case of Youtube. International Journal of Management Education, 16(1), 37-42.

https://doi.org/10.1016/j.ijme.2017.12.001

Ramírez-Ochoa, M. I. (2016). Posibilidades del uso educativo de YouTube. Ra Ximhai, 12(6), 537-546.

Reschly, A., \& Christenson, S. L. (2006a). Promoting successful school completion. En G. Bear \& K. Minke (Eds.), Children's needs-III: Development, prevention, and intervention (pp. 103-113). Bethesda, MD: National Association of School Psychologists.

Reschly, A., \& Christenson, S. L. (2006b). Research leading to a predictive model of dropout and completion among students with mild disabilities and the role of student engagement. Remedial and Special Education, 27, 276-292.

Rico Yate, J. P., Ramírez Montoya, M. S., \& Montiel Bautista, S. (2016). Desarrollo de la competencia oral del inglés mediante recursos educativos abiertos. Apertura, 8(1).

https://r.issu.edu.do/l?l=10597xrh

Rodríguez Villalobos, M. C., \& Fernández Garza, J. (2017). Uso del recurso de contenido en el aprendizaje en línea: YouTube. Apertura, 9(1), 22-31. https://doi.org/10.32870/ap.v9n1.1018.

Ros-Gálvez, A. \& Rosa-García, A. (2013). Vídeos docentes de Microeconomía: análisis de su impacto externo e interno. Historia y Comunicación Social, $18,75-84$.

https://doi.org/10.5209/rev_HICS.2013.v18.43949

Ruggiero, T. E. (2000). Uses and gratifications theory in the 21st century. Mass Communication \& Society, 3(1), 3-37. https://doi.org/10.1207/ S15327825MCS0301_02

Statista (2019). Hours of video upload to YouTube every minute as of May 2019. https://bit.ly/3k1dRo3

Semich, G. W., \& Copper, J. (2018). Instructional videos as ICT for teacher professional development: Transitioning from the traditional classroom to YouTube. In Teacher Training and Professional 
Development: Concepts, Methodologies, Tools, and Applications (pp. 1051-1065). IGI Global. https://doi.org/10.4018/978-1-5225-5631-2.ch048

Snelson, C. (2011). YouTube across the disciplines: A review of the literature. MERLOT Journal of Online Learning and Teaching, 7(1), 159-169.

https://r.issu.edu.do/l?l=10555H8x

Soukup, P. A. (2014). Looking at, with, and through YouTube. Communication Research Trends, 33(3), 3-34. https://r.issu.edu.do/l?1=10556HGs

Tan, E., \& Pearce, N. (2012). Open education videos in the classroom: Exploring the opportunities and barriers to the use of YouTube in teaching introductory sociology. Research in Learning Technology, 19, $125-133$.

https://doi.org/10.3402/ rlt.v19s1/7783

Taylor, L., \& Parsons, J. (2011). Improving Student Engagement. Current Issues in Education, 14(1). http://cie.asu.edu

Torres-Ramírez, M., García-Domingo, B., Aguilera, J., \& De La Casa, J. (2014). Video- sharing educational tool applied to the teaching in renewable energy subjects. Computers \& Education, 73,160-177.

https://doi.org/10.1016/j.compedu.2013.12.014

Yazzie-Mintz, E. (2007). Voices of students on engagement: A report on the 2006 High School Survey of Student Engagement. Bloomington: Center for Evaluation \& Education Policy, Indiana University. https://r.issu.edu.do/l?l=10557PaL

Yellepeddi, V., \& Roberson, C. (2016). The use of animated videos to illustrate oral solid dosage form manufacturing in a pharmaceutics course. American Journal of Pharmaceutical Education, 80(8), 1-10. http://dx.doi.org/10.5688/ajpe808141

Zabalza, I., Peña, B., Zalba, B., \& Marín, J. M. (2019). Uso de YouTube como herramienta educativa de apoyo a la docencia en termodinámica e ingeniería térmica. En IN-RED 2019. V Congreso de Innovación Educativa y Docencia en Red (pp. 40-51). Editorial Universitat Politècnica de València. shorturl.at/aivHR

\section{CÓMO CITAR:}

García-Béjar, L. (2021). Experiencias de engagement de aprendizaje de jóvenes universitarios mexicanos con Youtube. RECIE. Revista Caribeña de Investigación Educativa, 5(2), 23-37. https://doi.org/10.32541/recie.2021.v5i2.pp23-37 